\title{
Isolating the retrieval of imagined pictures during episodic memory: activation of the left precuneus and left prefrontal cortex
}

\author{
Brian Nils Lundstrom, ${ }^{\mathrm{a}, \mathrm{b}}$ Karl Magnus Petersson, ${ }^{\mathrm{a}, \mathrm{c}, \mathrm{d}}$ Jesper Andersson, ${ }^{\mathrm{a}}$ \\ Mikael Johansson, ${ }^{\mathrm{e}}$ Peter Fransson, ${ }^{\mathrm{a}}$ and Martin Ingvar ${ }^{\mathrm{a}, *}$ \\ a Department of Clinical Neuroscience, Karolinska Institutet, Stockholm, Sweden \\ ${ }^{\mathrm{b}}$ Medical Scientist Training Program, University of Washington, Seattle, Washington, USA \\ ${ }^{\mathrm{c}}$ Max Planck Institute for Psycholinguistics, Nijmegen, The Netherlands \\ ${ }^{\mathrm{d}}$ F.C. Donders Centre for Cognitive Neuroimaging, Katholieke Universiteit Nijmegen, The Netherlands \\ e Department of Psychology, Lund University; Lund, Sweden
}

Received 14 April 2003; revised 20 July 2003; accepted 23 July 2003

\begin{abstract}
The posterior medial parietal cortex and the left prefrontal cortex have both been implicated in the recollection of past episodes. In order to clarify their functional significance, we performed this functional magnetic resonance imaging study, which employed event-related source memory and item recognition retrieval of words paired with corresponding imagined or viewed pictures. Our results suggest that episodic source memory is related to a functional network including the posterior precuneus and the left lateral prefrontal cortex. This network is activated during explicit retrieval of imagined pictures and results from the retrieval of item-context associations. This suggests that previously imagined pictures provide a context with which encoded words can be more strongly associated.
\end{abstract}

(C) 2003 Elsevier Inc. All rights reserved.

\section{Introduction}

The ability to remember connects us with our world and past as well as potentially influences our future. In recent years, models of human memory have become increasingly sophisticated. Despite differing terminologies, one can fractionate the conscious, or explicit, aspect of this ability into three parts: semantic, working, and episodic memory (e.g., Schacter and Tulving, 1994; Eichenbaum and Cohen, 2001). Roughly speaking, semantic memory comprises general world knowledge, such as word meaning and word use; it stores, retrieves, and attaches this information to stimuli, for example, knowing the meaning and practical use of the word "lethean." Working memory refers to the short-term maintenance and manipulation of information during processing, such as remembering that lethean means "forgetful" long enough to integrate its meaning into a context.

Finally, episodic memory is employed for storage and

* Corresponding author.

E-mail address: martin@ingvar.com (M. Ingvar). recall of previous experiences or episodes, and it allows people to reflect upon their personal past, such as knowing from which personal experience their knowledge of lethean derived. Entailing more than just event memory and event recall, some have suggested that episodic memory necessarily includes a special awareness for subjective time known as autonoetic consciousness; it enables people to mentally travel backward in time and knowingly retrieve information from a given personal experience (e.g., Baddeley, 2001; Tulving 2002; Wheeler, 2000). In other words, episodic memory allows current knowledge to be associated with past experiences. This kind of memory is dependent upon retrieving contextual aspects, such as time and place, and is the focus of the current study.

Two kinds of memory tasks have commonly been used to test episodic memory in neuroimaging: item recognition and source memory (Rugg and Henson, 2003). Item recognition tasks commonly require an old/new decision to be made regarding a testing item; however, a feeling of familiarity may be sufficient for a correct answer even in the absence of specific recall of the encoding episode (e.g., 
Mandler, 1980). In contrast, source memory tasks demand explicit recall of contextual information, where subjects must remember not only an item but also the context within which it was presented. When testing source memory, retrieval contexts have consisted of the spatial or temporal location of dots, words, or pictures at presentation or the semantic manner in which words were encoded (e.g., Cansino et al., 2002; Dobbins et al., 2002; Henson et al., 1999; Nolde et al., 1998a; Nyberg et al., 1996; Ranganath et al., 2000; Rugg et al., 1999). Because one must distinguish between trial-specific aspects of recollection and familiarity, neither blocked designs nor a dependence on item recognition tasks can exhaustively examine episodic retrieval; however, an event-related design coupled with a source memory task, as used in the present study, offers an alternative (Rugg et al., 2002; Rugg and Wilding, 2000).

In an effort to further clarify aspects of episodic memory and the distinction between item and source memory, Johansson et al. (2002) conducted an EEG-based event-related potential (ERP) study, where words presented during encoding were accompanied either by a matching visual picture of the word or a matching image that the subject imagined. Two types of recognition tasks were then used. During old/new item recognition, only the word was of concern; however, during source memory the encoding context of the word, that is, the word-picture connection, assumed importance. Subjects were asked to remember the word and the type of associated picture, whether the picture had been viewed or imagined. This study found an old/new effect of earlier onset and greater magnitude for retrieved items that had been imagined rather than viewed. In addition, when compared with item recognition, the source memory task elicited a greater and longer response over prefrontal regions.

Using a paradigm similar to the one described above, this event-related functional MRI (fMRI) study examined the neural correlates of explicit source memory retrieval, where previously imagined items were expected to produce a more robust response than previously viewed items, as suggested by Johansson et al. (2002). When adapted to fMRI, the single, two-task retrieval session of this paradigm presents an opportunity to isolate the effects of episodic retrieval from the confounds of further encoding during the retrieval task, mixed correct and incorrect responses, and general task-specific effects (e.g., Rugg and Wilding, 2000). Given its associative and more complex nature, this study specifically addresses questions regarding two areas that have been consistently linked with episodic memory: the posterior medial parietal cortex (precuneus) and the prefrontal cortex (PFC).

In brief, activation of the posterior medial parietal cortex has been a ubiquitous finding in neuroimaging studies of episodic memory (e.g., Cabeza and Nyberg, 2000; Rugg and Henson, 2003). Activation of the precuneus has been proposed to play a crucial role in mental imagery used during recall, such as during imagery of concrete nouns (e.g.,
Grasby et al., 1993; Fletcher et al., 1995; Shallice et al., 1994). However, evidence suggests that the precuneus can be functionally disassociated into an anterior and posterior part and that the posterior precuneus rather plays a more general role in episodic retrieval (Buckner et al., 1996; Fletcher et al., 1998). A more recent study found posterior precunueal activation during a paired word associate memory task using both concrete and abstract nouns as well as two presentation modalities (auditory or visual) thereby suggesting a function in episodic memory that is independent of both the imagery content of words and differing presentation modalities (Krause et al., 1999). Thus, the precuneus has been implicated in episodic tasks employing associations of word pairs, whether abstract or concrete, and at least two presentation modalities. Here, we employ word-picture associations using two types of pictures as context.

The prefrontal cortex is clearly involved in aspects of memory encoding and retrieval, and lesion studies implicate the PFC in source memory (e.g., Shimamura, 1995). Neuroimaging studies have noted that source memory retrieval yields left-lateralized PFC activation and that learning-related decreases during source memory retrieval are leftdominant (Dobbins et al., 2002; Nolde et al., 1998a; Petersson et al., 2001). The left lateral PFC may allow and guide access of semantic knowledge (e.g., Friederici, 2002; Hagoort et al., 2002; Poldrack et al., 1999; Wagner et al., 2001). Other hypotheses suggest that the left lateral PFC can be expected during complex tasks of episodic retrieval and plays a more general role, possibly in selection of relevant information and inhibiting irrelevant information or specifying search parameters (Fletcher and Henson, 2001; Nolde et al., 1998b; Thompson-Schill et al., 1999). Here, we examine the PFC during an isolated aspect of source memory.

This study suggests more specific roles for the precuneus and PFC during episodic memory retrieval. Specifically, study design allows inferences to be made regarding contextual association since, during the source memory task but not the item recognition task, a conscious association between item and learning context is required. Results relate also to the retrieval differences of imagined versus viewed contextual information. Further, activation resulting from intertask (source memory minus item recognition) comparisons or those using different contextual information (imagined minus viewed) should be largely devoid of semantic retrieval effects, thereby separating the cerebral activation effects of episodic memory from semantic memory.

\section{Experimental procedures}

\section{Participants}

Twenty-one healthy, right-handed volunteers (10 female) with an average age of 24 years (range $20-28$, SD $=2.8$ ) participated in the study. All were prescreened, had no 
history of previous neurological conditions, and gave written informed consent. The ethics committee of Karolinska Hospital/Institutet approved the study protocol.

\section{Experimental stimuli}

From a pool of 120 common concrete nouns, 60 words were randomly selected and divided into sets of 10 words. These six sets were matched for word length (means 5.16.4 letters, range 3-10 letters) and frequency (means 3.85.6 per million, range $1-12$ per million). Words were selected and paired with corresponding black-and-white line drawings (Snodgrass and Vanderwart, 1980) as in Johansson et al. (2002). A one-word noun could easily describe each of these drawings, and this label was suitably concrete to evoke a corresponding mental image. Word sets were assigned to encoding and retrieval trial types such that material was counterbalanced temporally and with respect to stimulus type.

\section{Experimental design}

Volunteers participated in a word-picture association task that required one blocked fMRI encoding session (data not reported here) and one fMRI retrieval session comprising two blocks, one for each of the two event-related tasks (Fig. 1). During encoding, presented words were followed by either a visually perceived matching picture ("viewed" trial) or a blank screen prompting subjects to imagine a matching image ("imagined" trial). During retrieval two conditions were present: an item recognition task, where subjects answered "old" or "new," and a source memory task, where subjects answered "viewed," "imagined," or "new." For example, an imagined retrieval trial means that the subject had seen the word previously and had imagined a picture with that word. Three of the stimuli word sets were used for the item recognition task (old viewed, old imagined, and new trials), and three of the word sets were used for the source memory task (viewed, imagined, and new trials).

Each subject participated in one encoding and one retrieval session. Sessions lasted approximately $8 \mathrm{~min}$ each and were separated by 1-2 min when the subject counted backward from 100 by 3 . Subject responses were recorded via a finger-response pad. The first (thumb), second, and third fingers were used for the 3-point rating during encoding, the two-choice item recognition task (first and second fingers only), and the three-choice source memory task. All subjects trained outside the camera before MR scanning and reported excellent understanding of the instructions in a postexperiment interview.

\section{Encoding}

Blocked encoding consisted of four trial types: viewed, viewed baseline, imagined, and imagined baseline, where "viewed" or "imagined" refers to whether the picture of the
A

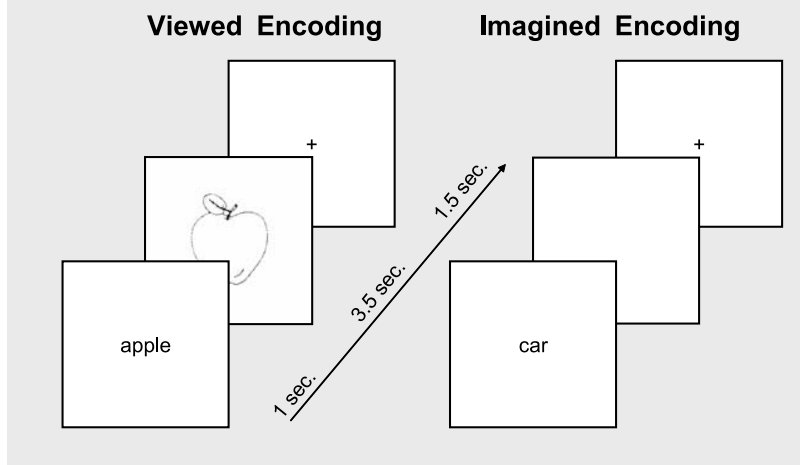

B

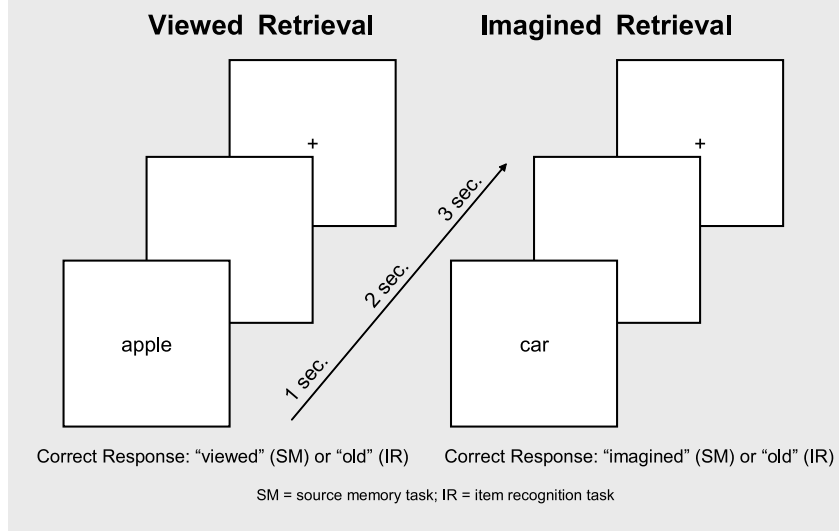

Fig. 1. Experimental design of blocked encoding and event-related retrieval sessions. (A) Blocked encoding consisted of viewed and imagined trials, where subjects viewed a word and either viewed a corresponding picture or imagined a corresponding mental image. (B) Retrieval consisted of an event-related source memory task and an event-related item recognition task; trial types appeared the same to subjects, but answering instructions differed. For this example, during source memory correct answers would be "viewed" and "imagined," respectively. However, during item recognition correct answers would be "old" and "old," respectively. New words and baseline words were intermixed during retrieval.

word-picture pair was visually perceived or imagined (Fig. 1). Words were presented for $1 \mathrm{~s}$ followed by $3.5 \mathrm{~s}$ to view or imagine a picture; a fixation cross appeared for $1.5 \mathrm{~s}$ between trials. For viewed trials, an accompanying blackand-white picture followed the presented word; for imagined trials, a blank screen followed the presented word. Subjects were asked to subjectively rate their affection toward the viewed or imagined picture on a 3-point scale. For baseline trials, presented words were either "Knapp1," "Knapp2," or "Knapp3," where knapp is translated as button from Swedish; subjects responded according to the number following "knapp." During viewed baseline, a nonfigurative picture followed the presented word; subjects were instructed to view these pictures passively. During imagined baseline, a fixation cross followed the presented baseline word. 
Encoding trials were presented as 33-s blocks ( 5 trials + 3 -s instructions), which were temporally balanced within sessions and counterbalanced across sessions and subjects. Encoding sessions included 16 blocks consisting of 40 encoded words and 40 baseline trials.

\section{Retrieval}

Each event-related retrieval session included two tasks: an item recognition task (old/new) and a source memory task (viewed/imagined/new). Each of the two tasks contained four trial types for a total of 8 retrieval trial types. Words were presented for $1 \mathrm{~s}$ followed by $2 \mathrm{~s}$ of a blank screen. Subjects were required to answer within this 3-s period; they were instructed to answer as quickly as possible but without accidentally choosing incorrectly. Three seconds of fixation cross separated every trial.

All words were presented a pseudorandom manner such that each of the four trial types (viewed, imagined, new, baseline) were balanced temporally and were presented an equal number of times both midway and at the end of each retrieval section. Each retrieval task was preceded by one 6-s instruction screen and employed 20 old words, 10 new words, and 10 baseline words ("Knapp\#"). During baseline trials, subjects answered according to the number following knapp; source memory retrieval employed Knapp1, Knapp2, and Knapp3, while item recognition used only Knapp1 and Knapp2. Each retrieval session consisted of a source memory and item recognition task. The presented order of the retrieval tasks was balanced across subjects.

\section{fMRI procedure}

A GE Signa 5. $\times 1.5-\mathrm{T}$ scanner was employed to obtain T2*-weighted echo-planar images (acq. matrix $64 \times 64$, voxel $3.5 \times 3.5 \times 3.4 \mathrm{~mm}$, TE $40 \mathrm{~ms}$, TR 4.2); 42 slices were acquired in consecutive, ascending order with $3-\mathrm{mm}$ slice thickness and 0.4-mm interslice spacing. One hundred twenty-six volumes were acquired during the encoding session, and 118 volumes during the retrieval session. Visual information was presented via back-projection onto a screen, which was viewed by subjects using a binocularmirror apparatus attached to the head coil.

\section{fMRI data analysis and modeling}

Image preprocessing was performed using SPM99 software (Wellcome Dept. of Neurology, UK; http://www.fil. ion.ucl.ac.uk). All statistical modeling was performed using the general linear model (GLM) as implemented in SPM99 (Friston et al., 1995).

\section{Preprocessing}

For each subject, images were realigned to the first volume and resampled using truncated sinc interpolation. Slice timing correction to the middle slice was performed for event-related (retrieval) sessions. T1-weighted anatomical image volumes were coregistered to the mean $\mathrm{T} 2 *$-weighted echo planar image (EPI), and spatially normalized to an approximate Talairach space (Talairach and Tournoux, 1988) as defined by the SPM99 MNI T1 template. The transformation parameters thus obtained were applied to the EPI time series, using trilinear interpolation. Finally, images were smoothed using a 12-mm FWHM isotropic Gaussian filter.

\section{Retrieval sessions: first level}

Trial lengths were considered to be the time between the onset of word presentation and subject response, i.e., when the subject pressed a button. Every trial type was modeled as a series of variable length boxcars convolved with the SPM99's default canonical HRF. Additionally, temporal derivatives of the trial types were modeled as separate regressors. Nonbaseline events were sorted into correct (e.g., saying a word was old when it was), incorrect (e.g., saying a word was new when in fact it had been seen before), and failure to answer (e.g., when the subject did not answer within $3 \mathrm{~s}$ ) responses. Eight correct and eight incorrect trial types yielded a total of 16 distinct "event-types" plus event instructions that were all modeled separately. Linear contrasts of parameter estimates for use in a secondlevel analysis included regressors for correct responses only. Data were high-pass-filtered (range 34-96 s) and low-pass-filtered (Gaussian 4 s FWHM).

\section{Retrieval comparisons}

Parameter estimates were compared in three general ways: within the source memory task, between the source memory and item recognition tasks, and within the item recognition task. Given the results of Johansson et al. (2002) regarding imagined vs viewed and the source memory vs item recognition tasks and the desire to isolate aspects of source memory, comparisons of this study center on the source memory (SM) imagined trials, where this trial type may give the most robust signal. A total of eight trial types were used and are as follows: SM viewed, SM imagined, SM new, SM baseline, item recognition (IR) viewed, IR imagined, IR new, and IR baseline. Comparisons are as follows:

(1) SM imagined vs SM new

(2) (SM imagined - SM baseline) vs (IR imagined IR baseline)

(3) SM imagined vs SM viewed

(4) SM old/new effect vs IR old/new effect

(5) SM viewed vs SM new

(6) IR imagined vs. IR new

(7) IR viewed vs IR new

(8) (SM new - SM baseline) vs (IR new - IR baseline)

Comparison (1) examines imagined retrieval success, while comparisons (2) and (3) allow the explicit retrieval of context and context type to be isolated, respectively. Fur- 
Table 1

Source memory (SM) and item recognition (IR) activation

\begin{tabular}{|c|c|c|c|c|c|c|}
\hline & Area & $x, y, z(\mathrm{~mm})$ & Brodmann area & $Z$ score & Voxel $P$ corr. & Cluster $P$ corr. \\
\hline \multirow{11}{*}{$\begin{array}{l}\text { SM imagined vs SM new } \\
\text { (Fig. 2A) }\end{array}$} & $\mathrm{L}$ precuneus, parietal cx & $-6,-64,30$ & $7,19,29,30,31,39,40$ & 5.54 & 0.001 & 0.000 \\
\hline & $\mathrm{L}$ posterior precuneus & $-6,-70,36$ & 7 & 5.37 & 0.003 & \\
\hline & $\mathrm{L}$ parietal $\mathrm{cx}$ & $-48,-52,44$ & 39,40 & 4.93 & 0.02 & \\
\hline & $\mathrm{R}$ lateral parietal cx & $34,-70,40$ & $7,19,40$ & 5.04 & 0.013 & 0.010 \\
\hline & L lateral prefrontal $\mathrm{cx}$ & $-42,40,12$ & $10,44,45,46,47$ & 4.66 & 0.058 & 0.000 \\
\hline & intracluster maxima & $-38,52,4$ & 10 & 4.63 & 0.064 & \\
\hline & & $-38,34,26$ & 9 & 4.59 & 0.076 & \\
\hline & & $-44,48,8$ & 10 & 4.54 & 0.091 & \\
\hline & & $-46,28,20$ & 45,46 & 4.42 & 0.137 & \\
\hline & & $-42,18,5$ & 45,47 & 4.24 & 0.241 & \\
\hline & & $-26,54,18$ & 10 & 3.33 & 0.983 & \\
\hline \multirow{3}{*}{$\begin{array}{l}\text { SM imagined vs. IR imagined } \\
\quad \text { (Fig. 2B) }\end{array}$} & L lateral prefrontal $\mathrm{cx}$ & $-58,18,14$ & 44,45 & 4.58 & 0.072 & 0.012 \\
\hline & L posterier precuneus & $-12,-64,46$ & 7 & 4.53 & 0.086 & 0.068 \\
\hline & $\mathrm{R}$ cingulate gyrus & $12,-48,-2$ & 30 & 3.98 & 0.446 & 0.017 \\
\hline \multirow{2}{*}{$\begin{array}{l}\text { SM imagined vs. SM viewed } \\
\quad(\text { Figs. } 2 \mathrm{C} \text { and } \mathrm{D})\end{array}$} & L lateral prefrontal $\mathrm{cx}$ & $-48,26,24$ & $44,45,46$ & 3.96 & 0.454 & 0.035 \\
\hline & L posterior precuneus & $-18,-66,40$ & 7 & 3.93 & 0.488 & 0.116 \\
\hline $\begin{array}{l}\text { SM old/new vs IR old/new } \\
\text { (Fig. 3) }\end{array}$ & $\mathrm{L}$ ant insula, frontal opcm & $-40,20,8$ & 44,45 & 4.54 & 0.082 & 0.019 \\
\hline SM viewed vs SM new & $\mathrm{L}$ posterior precuneus & $-10,-68,26$ & 7 & 3.53 & 0.88 & 0.120 \\
\hline IR viewed vs IR new & $\mathrm{L}$ posterior precuneus & $-2,-64,42$ & 7 & 3.69 & 0.752 & 0.091 \\
\hline
\end{tabular}

Note. Comparisons were performed between source memory and item recognition, within source memory, and within item recognition retrieval tasks. Abbreviations: ant, anterior; cx, cortex; L, left; opcm, operculum; R, right.

ther, comparison (4) may suggest difference in SM retrieval success vs IR retrieval success. As in Johansson et al. (2002), the other three old/new effects of comparisons (5)(7) were examined. Finally, comparison (8) was examined, as it would display memory effects independent of retrieval success.

\section{Retrieval sessions: second level}

Images constituting subject-specific linear combinations of the parameters obtained at the first level, e.g., contrasts spanning parameter estimates for each individual subject, were created. These 21 images, one for every subject, were entered into one second-level model (a one-sample $t$ test) for each contrast, yielding a random effects model (Holmes and Friston, 1998).

\section{Inference}

All inferences were based on the SPM $\{\mathrm{t}\}$ values from the second-level analysis. SPM $\{\mathrm{t}\}$ values were thresholded at the $P<0.001$ uncorrected $(t=3.55$ ) level; the number and size of connected clusters of suprathreshold voxels were recorded. Corrected (for multiple nonindependent comparisons) cluster-level $P$ values were calculated for each cluster based on their spatial extent (Friston et al., 1994). Clusters with a corrected $P$ value less than 0.1 , which yielded $\sim 150$ voxels, were considered significant. In addition, given our focused interest on the medial posterior parietal cortex, two precuneal areas with cluster $P$ values of 0.116 and 0.120 were examined due to the activation of this area in other second-level contrasts (Table 1) and in other episodic memory studies.

\section{Results}

\section{Behavioral performance}

Overall, subjects were more accurate during new trials than during old trials, and reaction times during the source task were longer than reaction times during the item recognition task (Table 2). During retrieval, accuracy was $90.00 \%$ $(\mathrm{SD}=9.72)$ for source memory, $93.02 \%(\mathrm{SD}=6.40)$ for item recognition, and $91.51 \%(\mathrm{SD}=6.50)$ overall. Regarding accuracies, a two-way ANOVA employing factors of task and item type demonstrated a marginal main effect of item type $(F(2,120)=3.0, P=0.055)$ but did not demonstrate a main effect of task $(F(1,120)=2.2, P=0.141)$ or interaction of task and item type $(F(2,120)=0.5, P=$ $0.619)$. New trials showed a greater accuracy when compared to old trials (paired $t$ test, $T(40)=2.4011, P=0.011$ ). Regarding reaction times, a two-way ANOVA employing factors of task (source memory vs item recognition) and item type (viewed vs imagined vs new) demonstrated a strong main effect of task $(F(1,120)=21.7, P=0.00005)$ but no main effect of item type $(F(2,120)=0.1, P=0.378)$ or interaction of task and item type $(F(2,120)=2.2, P=$ $0.115)$.

In addition to subject accuracy, an IR index was calculated for viewed and imagined items in both the source memory and the item recognition tasks. This IR index ("cor- 
Table 2

Reaction times and accuracy for each of the six nonbaseline trials involved in retrieval

\begin{tabular}{|c|c|c|c|c|c|c|c|c|c|c|}
\hline \multirow[b]{2}{*}{ Source memory } & \multicolumn{4}{|c|}{ Viewed } & \multicolumn{4}{|c|}{ Imagined } & \multicolumn{2}{|c|}{ New } \\
\hline & $\begin{array}{c}\text { RT } \\
1540 \\
(294)\end{array}$ & $\begin{array}{l}\% \text { Correct } \\
87.14 \\
(12.70)\end{array}$ & $\begin{array}{l}\text { IR index } \\
0.93 \\
(0.11)\end{array}$ & $\begin{array}{l}\text { SM index } \\
0.85 \\
(0.16)\end{array}$ & $\begin{array}{c}\text { RT } \\
1720 \\
(327)\end{array}$ & $\begin{array}{l}\% \text { Correct } \\
89.05 \\
(13.38)\end{array}$ & $\begin{array}{l}\text { IR index } \\
0.92 \\
(0.11)\end{array}$ & $\begin{array}{l}\text { SM index } \\
0.88 \\
(0.15)\end{array}$ & $\begin{array}{c}\text { RT } \\
1467 \\
(344)\end{array}$ & $\begin{array}{l}\% \text { Correct } \\
93.81 \\
(11.17)\end{array}$ \\
\hline & \multicolumn{4}{|c|}{ Viewed } & \multicolumn{4}{|c|}{ Imagined } & \multicolumn{2}{|c|}{ New } \\
\hline Item recognition & $\begin{array}{c}\text { RT } \\
1276 \\
(386)\end{array}$ & $\begin{array}{l}\% \\
92 . \\
(9 .\end{array}$ & rect & $\begin{array}{l}\text { IR index } \\
0.90 \\
(0.11)\end{array}$ & $\begin{array}{c}\mathrm{RT} \\
1266 \\
(370)\end{array}$ & $\begin{array}{l}\text { \% Correct } \\
90.00 \\
(14.14)\end{array}$ & & & $\begin{array}{c}\text { RT } \\
1326 \\
(339)\end{array}$ & $\begin{array}{l}\text { \% Correct } \\
96.19 \\
(5.90)\end{array}$ \\
\hline
\end{tabular}

Note. Reaction times are displayed in milliseconds and accuracy in percentages. Standard deviations are in parentheses. The Item Recognition (IR) Index is calculated as the fraction of correct old responses minus false alarms, while the Source Memory (SM) Index is the fraction of correct source attributions minus the source specific false alarms.

rect old responses" minus "false alarms") was based on all correct "old" judgments, regardless of whether the source attribution was correct. Further, a SM index was calculated for items in the source memory task, and this SM index ("correct source attributions" minus "source-specific false alarms") accounted for discrimination of source. A two-way ANOVA employing factors of task (source memory vs item recognition) and item type (viewed vs imagined) for the IR indices did not display a main effect of task $(F(1,80)=1.6$, $P=0.205)$ or item type $(F(1,80)=0.5, P=0.467)$, or an interaction of task and item type $(F(1,80)=0.1, P=$ $0.716)$. The SM indices were likewise not statistically different $(F(1,40)=0.3, P=0.560)$.

\section{Imaging data}

In general, data of episodic retrieval were markedly leftlateralized with, for example, no instances of significant right PFC activation. (Strong bilateral activation was seen in baseline comparisons.) However, due to the relatively small number of trials per subject, these data may underestimate activation of episodic retrieval. Encoding data were not considered in this study.

In general, the comparison of SM imagined vs SM new (Fig. 2A) examines a specific retrieval success that presumably comprises both the recognition for a studied word and the explicit knowledge that an imagined picture was encoded with that word. However, the dependence of this retrieval success on the explicit retrieval of source is not clear; thus we test SM imagined vs IR imagined with appropriate baseline trials subtracted (Fig. 2B). During IR imagined trials, even though source information may be recalled, we expect that its retrieval is either tentative or implicit. Further, the source information retrieved during SM imagined trials is source-specific and may differ from viewed source information, although we expect the imagined source to yield a stronger signal, thus SM imagined vs SM viewed (Fig. 2C). Finally, in an attempt to characterize the difference between intertask retrieval success on the whole, we compare the old/new effects of each task (Fig. 3).
As expected from the results of Johansson et al. (2002), SM imagined trials minus SM new trials displays a robust old/new effect yielding large areas of activation in the medial and left lateral parietal regions and the left lateral $\mathrm{PFC}$, and a smaller area in the right lateral parietal cortex (Fig. 2A; table 1). In order to examine the effects of explicit context retrieval, IR imagined trials were subtracted from SM imagined trials, and activation was seen in the posterior precuneus and left lateral PFC (Fig. 2B; Table 1). The effects of retrieval due to encoding context type were examined via the SM imagined vs SM viewed comparison, showing similar activation in the posterior precuneus and left lateral PFC (Figs. 2C and D; Table 1). As readily calculated from Table 1, local maxima in the posterior precuneus were within $14 \mathrm{~mm}$ for each of these three contrasts, whereas the left PFC cluster for the SM imagined minus IR imagined comparison was approximately $16 \mathrm{~mm}$ posterior and lateral to maxima in the other two comparisons, which were themselves less than $5 \mathrm{~mm}$ apart (Table 1).

When compared with item recognition, Johansson et al. (2002) found that source memory was associated with a mean prefrontal ERP old/new effect (i.e., SM imagined plus SM viewed minus SM new trials) of greater magnitude and longer duration. Therefore, we compared the two old/new effects of source memory with those of item recognition and found a significant activation in the anterior insula and frontal operculum (Fig. 3, Table 1). This activation is primarily due to the old/new effects of source memory (Fig. $3 B)$. Hence, the mean old/new effect of source memory contributes to this left prefrontal activation more than the mean old/new effect of item recognition. Finally, given the study of Johansson et al. (2002), specific old/new effects for SM viewed, IR imagined, and IR viewed trials were also considered. For the comparisons SM viewed vs SM new and IR viewed vs IR new, only activation in the left posterior precuneus was significant (Table 1; the former of these two activations was marginally significant). No significant activation was seen in the IR imagined vs IR new or SM new vs IR new comparison. 


\section{A. SM Imagined $>$ SM New}

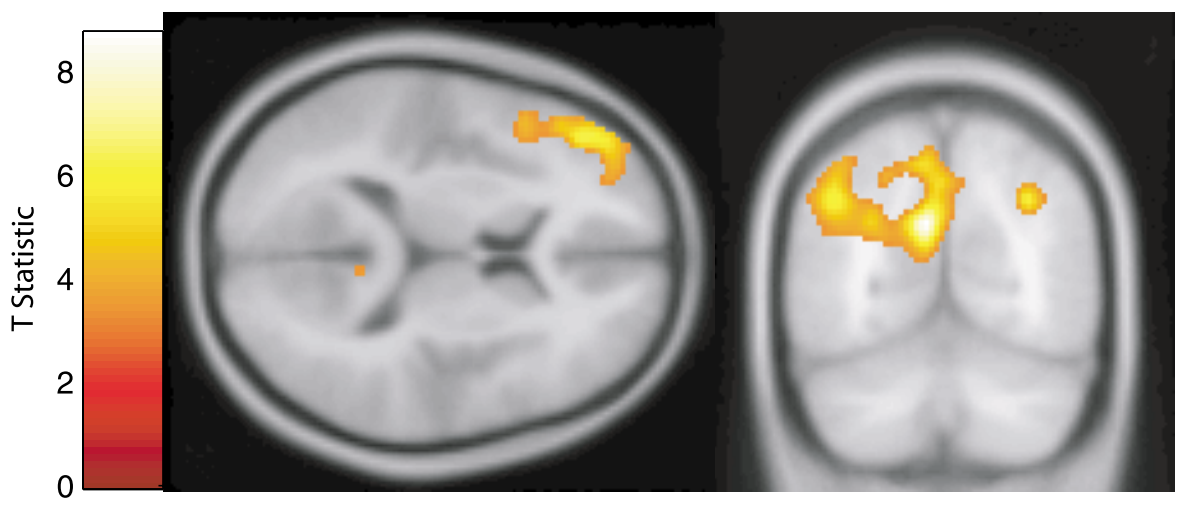

B. SM Imagined > IR Imagined

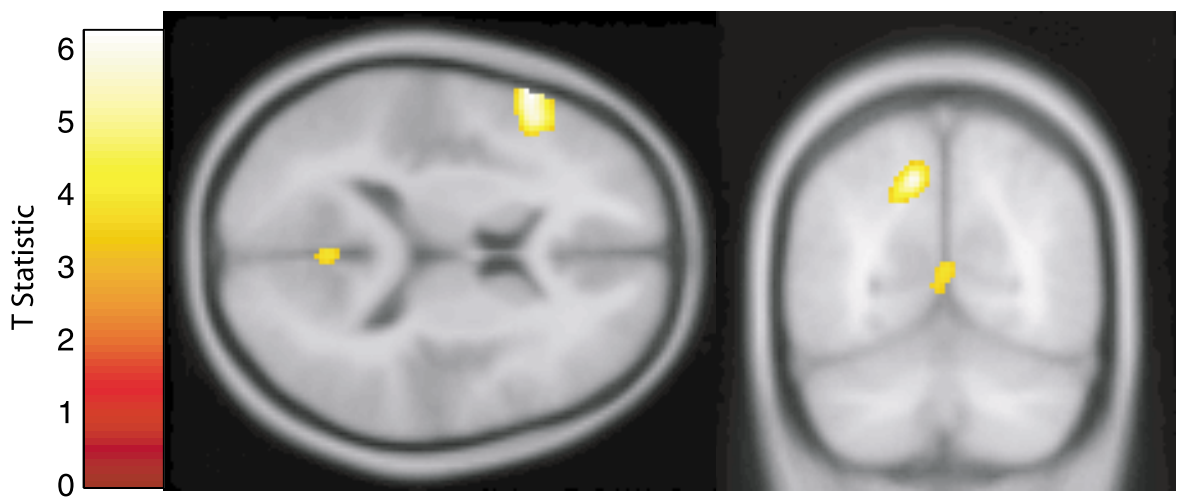

\section{SM Imagined > SM Viewed}

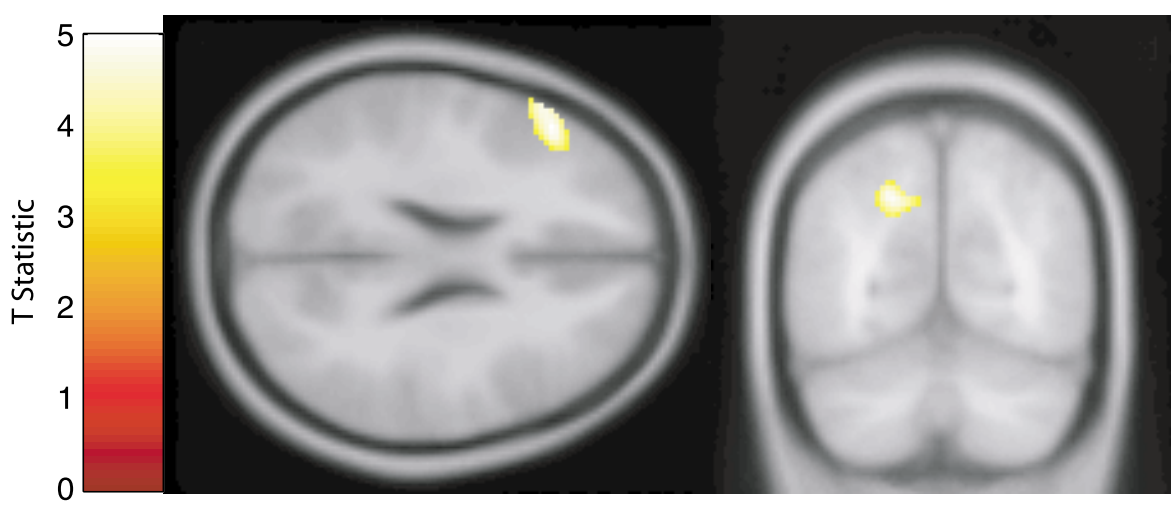

D. SM Imagined > SM Viewed

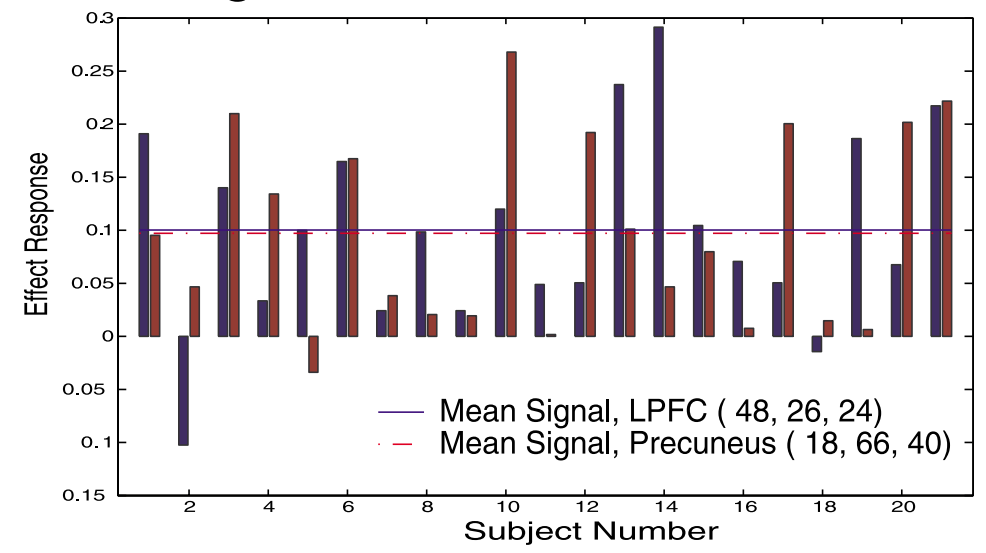




\section{A. SM Old/New > IR Old/New}

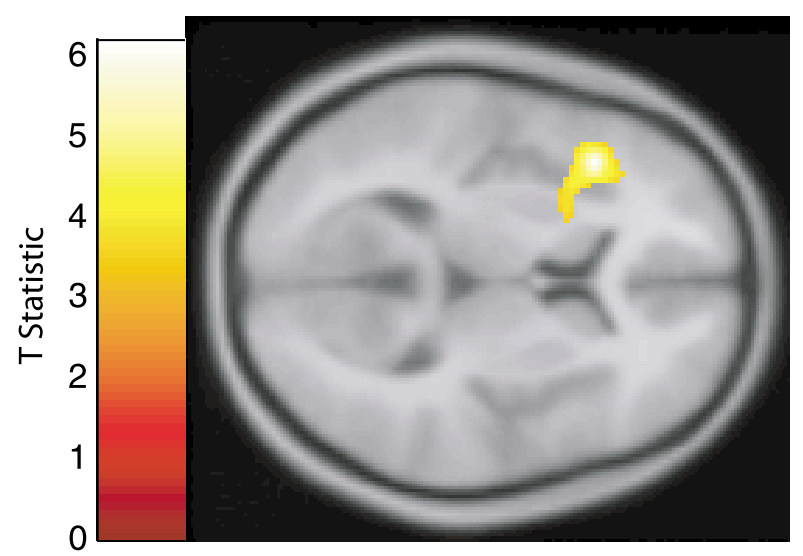

\section{B. Individual Old/New Effects}

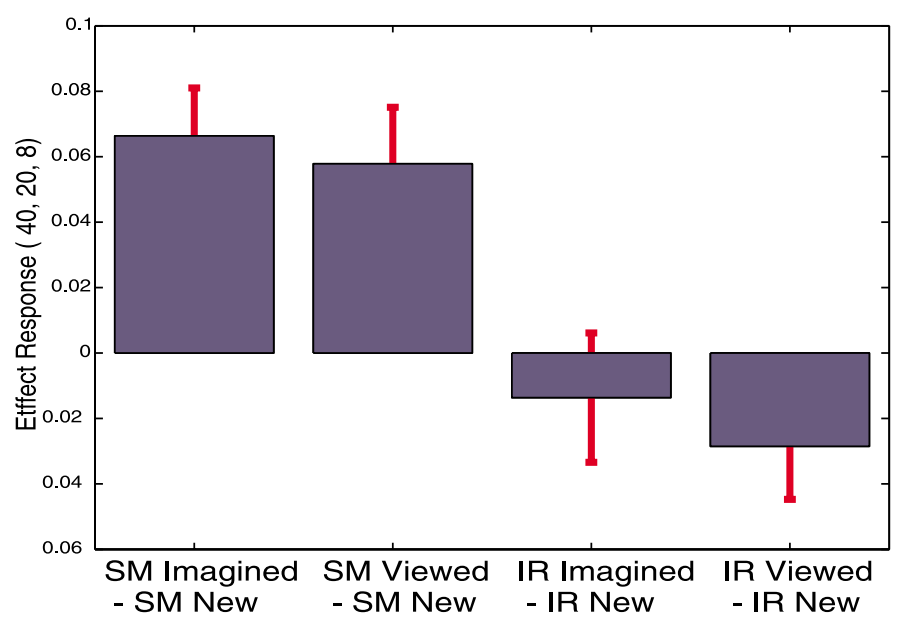

Fig. 3. Anterior insula and frontal operculum activation thresholded at $P<0.001(t=3.55)$ with an extent greater than $\sim 150$ voxels displayed on an axial anatomical slice with an accompanying graph showing the contribution of individual old/new effects to this comparison. (A) Source memory old/new effect minus item recognition old/new effect, where each old/new effect comprises old trials (viewed and imagined) minus new trials. (B) Parameter estimates for each of the four old/new effects contributing to the activation at its peak coordinate $(-40,20,8)$.

\section{Discussion}

Data suggest that the posterior precuneus activates during retrieval due to explicit revival of contextual associations (Fig. 2), that the left lateral prefrontal cortex is more active during source memory than item recognition (Fig. 2B) and during retrieval of imagined rather than viewed contexts (Fig. 2C), and that a left-lateralized network consisting of these two regions is involved in complex episodic retrieval as isolated from effects of semantic retrieval.

\section{Medial posterior parietal cortex}

Previously, activation of the precuneus has been proposed to play a crucial role in the mental imagery of episodic retrieval (e.g., Grasby et al., 1993; Fletcher et al., 1995; Shallice et al., 1994). However, evidence suggests that the functional role of the precuneus may need to be elaborated, and more recent evidence suggests that the precuneus plays a more general role in episodic retrieval and can be functionally disassociated into an anterior and posterior part (Buckner et al., 1996; Fletcher et al., 1998). For example, in a more recent study, posterior precuneal activation was observed during a paired word associate memory task employing both concrete and abstract nouns as well as two different presentation modalities (auditory or visual), thus suggesting a function in episodic memory that is inde- pendent both of the imagery content of words and of differing presentation modalities (Krause et al., 1999).

Current results (Fig. 2) show that the posterior precuneus is activated during the explicit retrieval of source information and that it also appears to be preferentially activated by an imagined rather than a visually perceived context. Given the previously noted activation during word-pair association and here during what is essentially word-picture association, the posterior precuneus may activate during regeneration of previous contextual associations. Its apparent preferential activation during retrieval of an imagined context may represent the revival of relatively stronger associations. This is in agreement with the noted generation effect, where self-generated associations are remembered more accurately than perceived associations (e.g., Lockhart, 2000); it has also been noted that self-generation of a target word strengthens association with its context (Marsh et al., 2001).

In addition to the comparisons in Fig. 2, the posterior precuneus was also activated during SM viewed minus SM new and IR viewed minus IR new comparisons. These old/new activations suggest that the posterior precuneus is sensitive to the quality or amount of information retrieved, as previous studies also suggest (Nyberg et al., 2000; Rugg et al., 1998). However, no significant activation was seen in the IR imagined minus IR new comparison. One possible explanation is that subjects retrieved the imagined contexts less readily than the viewed pictures when they were not

Fig. 2. Left PFC and left posterior precuneal activations thresholded at $P<0.001(t=3.55)$ with an extent greater than $\sim 150$ voxels displayed on axial and coronal anatomical slices. (A) Source memory imagined minus source memory new; transverse $z=12$; coronal $y=-64$. (B) Source memory imagined minus item recognition imagined (with appropriate baseline trials subtracted); transverse $z=14$; coronal $y=-64$. (C) Source memory imagined minus source memory viewed; transverse $z=24$; coronal $y=-66$. (D) Parameter estimates for each subject during source memory imagined minus source memory viewed with means displayed as horizontal lines. 
specifically asked to consciously retrieve them, such as during item recognition. This could be due to less informational content in the imagined pictures in accordance with the idea that self-generated events generally contain less perceptual and spatiotemporal information than perceived events (e.g., see discussion of Marsh et al., 2001). In addition, ERP studies have supported the notion that parietal old/new effects increase in a graded fashion with the amount or quality of information retrieved (e.g., Wilding, 2000).

\section{Left prefrontal cortex}

It has been suggested that complex episodic memory tasks require left PFC-mediated processes, and our results support this conclusion (Nolde, 1998b, Fig. 2). Both neuroimaging and ERP studies employing source memory retrieval have also found left-lateralized PFC activation (Dobbins et al., 2002; Nolde et al., 1998a; Ranganath et al., 2000; Ranganath and Paller, 1999, 2000; Rugg et al., 1999). Specifically, in an attempt to parse the prefrontal processes of source memory, Dobbins et al. identified regions in the left PFC relating to lexical/phonological maintenance, controlled semantic analysis/cue specification, and monitoring/ evaluating recollections, where these regions roughly corresponded to the posterior inferior frontal gyrus, anterior inferior frontal gyrus, and the posterior middle frontal gyrus, respectively.

In our study, the left PFC activation that resulted from the comparison of SM imagined minus IR imagined trials (Fig. 2B, $-58,18,14$ ) is roughly in the superior part of the anterior inferior frontal gyrus, an area specified by Dobbins et al. (2002) as cue specification, which is the matching of retrieval cue to characteristics of potential information being retrieved. This is then as expected when comparing source memory and item recognition. The other two left PFC activations of our study (Fig. 2A and B, $\sim-46,26,20$ ) occupy an area generally anterior to the posterior inferior frontal gyrus. If retrieval success does not affect the three processes of lexical maintenance, cue specification, and monitoring (i.e., they are task specific), as suggested by Dobbins et al. (2002), we would not expect activations that result from intratask source memory comparisons to subserve these processes. Rather, activation of this area may be related to item-specific processes important for the retrieval of contextual associations.

Motivated by Johansson et al. (2002), examining intertask old/new effects may suggest differences between the retrieval success of each task. When comparing the old/new effects of source memory with those of item recognition, we observed activation of the anterior insula and frontal operculum. Previous studies have noted activation of the frontal operculum bilaterally during a source memory and item recognition comparison (Rugg et al., 1999) and left-lateralized activation during an incorrect vs correct source memory comparison (Takahashi et al., 2002). It was suggested that the left-lateralized activation of the frontal operculum may reflect effort to retrieve source information (Takahashi et al., 2002), and our results would be consistent with this notion.

\section{Left prefrontal/precuneal network}

This experiment gives an example where the left-lateralized posterior precuneus and lateral PFC are engaged in tandem during episodic retrieval. The common thread in the three primary comparisons (Fig. 2) of our results is an item-related effect associated with source memory retrieval of an imagined picture. In addition to the left PFC proposed to be involved in complex episodic retrieval (Nolde et al., 1998b), other source memory studies have found posterior precuneal activation (e.g., Dobbins et al., 2002). Reflective processes of episodic memory may then include those mediated not only by the left lateral PFC but also by the posterior precuneus.

\section{Conclusions}

We suggest that the precuneus activates during regeneration of contextual associations, such as needed during source memory. In addition, these data are consistent with the view that a context comprising an imagined picture provides a stronger, although perhaps less vivid, contextword association than does a visually perceived picture. Further, the left lateral PFC appears to mediate the processing of this contextual information. The activation of the left PFC and posterior precuneus may then be a hallmark of rich, personal memory that is dependent on retrieval of source information.

\section{Acknowledgments}

Grants from the Swedish Medical Research Council (8276), the Karolinska Institutet, the Swedish Medical Association, the Knut and Alice Wallenberg Foundation, and the Family Hedlund Foundation supported this work. B.N.L. was supported by a fellowship from the Fulbright Commission in Sweden. We thank Guillen Fernandez, Rik Henson, Lars Nyberg, and Ivan Toni for comments on early versions of the manuscript as well as Alex Huk and Michael Shadlen for conversation and support.

\section{References}

Baddeley, A., 2001. The concept of episodic memory. Phil. Trans. R. Soc. Lond. B 356, 1345-1350.

Buckner, R.L., Raichle, M.E., Miezin, F.M., Petersen, S.E., 1996. Functional anatomic studies of memory retrieval for auditory words and visual pictures. J. Neurosci. 16 (19), 196-235. 
Cabeza, R., Nyberg, L., 2000. Imaging cognition II: an empirical review of 275 PET and fMRI studies. J. Cogn. Neurosci. 12 (1), 1-47.

Casino, S., Maquet, P., Dolan, R.J., Rugg, M.D., 2002. Brain activity underlying encoding and retrieval of source memory. Cereb. Cortex Mon. 12, 1048-1056.

Dobbins, I.G., Foley, H., Schacter, D.L., Wagner, A.D., 2002. Executive control during episodic retrieval: multiple prefrontal processes subserve source memory. Neuron 35, 989-996.

Eichenbaum, H., Cohen, N.J., 2001. From Conditioning to Conscious Recollection: Memory Systems of the Brain. Oxford University Press, New York.

Fletcher, P.C., Frith, R.S.J., Baker, S.C., Shallice, T., Frackowiak, R.S.J., Dolan, R.J., 1995. The mind's eye-precuneus activation in memoryrelated imagery. NeuroImage 2, 195-200.

Fletcher, P.C., Henson, R.N.A., 2001. Frontal lobes and human memory: insights from neuroimaging. Brain 124, 849-881.

Fletcher, P.C., Shallice, T., Frith, C.D., Frackowiak, R.S.J., Dolan, R.J., 1998. The functional roles of prefrontal cortex in episodic memory: II. Retrieval. Brain 121, 1249-1256.

Friederici, A.D., 2002. Towards a neural basis of auditory sentence processing. Trends Cogn. Sci. 6, 78-84.

Friston, K.J., Fletcher, P., Josephs, O., Holmes, A., Rugg, M.D., Turner, R., 1998. Event-related fMRI: characterizing differential responses. NeuroImage 7, 30-40.

Friston, K.J., Holmes, A.P., Worsley, K.J., Poline, J.B., Frith, C.D., Frackowiak, R.S.J., 1995. Statistical parametric maps in functional imaging: A general linear approach. Human Brain Mapp. 2, 189-210.

Friston, K.J., Worsley, K.J., Frackowiak, R.S.J., Mazziotta, J.C., Evans, A.C., 1994. Assessing the significance of focal activations using their spatial extent. Human Brain Mapp. 1, 210-220.

Grasby, P.M., Frith, C.D., Friston, K.J., Bench, C., Frackowiak, R.S.J., Dolan, R.J., 1993. Functional mapping of brain areas implicated in auditory-verbal memory function. Brain 116, 1-20.

Hagoort, P., Hald, P., Petersson, K.M., 2002. Semantic vs world knowledge integration during sentence comprehension, J. Cogn. Neurosci. Suppl., 159.

Henson, R.N.A., Shallice, T., Dolan, R.J., 1999. Right prefrontal cortex and episodic memory retrieval: a functional MRI test of the monitoring hypothesis. Brain 122, 1367-1381.

Holmes, A.P., Friston, K.J., 1998. Generalisability, random effects and population inference. NeuroImage Suppl. 7 (4), S754.

Johansson, M., Stenberg, G., Lindgren, M., Rosen, I., 2002. Memory for perceived and imagined pictures-an event-related potential study. Neuropsychologia 40, 986-1002.

Krause, B.J., Schmidt, D., Mottaghy, F.M., Taylor, J., Halsband, U., Herzog, H., Tellmann, L., Mueller-Gaertner, H.-W., 1999. Episodic retrieval activates the precuneus irrespective of the imagery content of word pair associates: a PET study. Brain 122, 255-263.

Lockart, R.S., 2000. Methods of memory research, in: Tulving, E., Craik, F.I.M. (Eds.), The Oxford Handbook of Memory, Oxford University Press, Oxford, pp. 597-608.

Mandler, G., 1980. Recognising: the judgement of previous occurrence. Psychologic. Rev. 87, 252-271.

Marsh, E.J., Edelman, G., Bower, G.H., 2001. Demonstration of a generation effect in context memory. Memory Cognition 29 (6), 798-805.

Nolde, S.F., Johnson, M.K., D’Esposito, M., 1998a. Left prefrontal activation during episodic remembering: an event-related fMRI study. Neuroreport 9, 3509-3514.

Nolde, S.F., Johnson, M.K., Raye, C.L., 1998b. The role of prefrontal cortex during tests of episodic memory. Trends Cogn. Sci. 2 (10), 399-406.

Nyberg, L., McIntosh, A.R., Cabeza, R., Habib, R., Houle, S., Tulving, E., 1996. General and specific brain regions involved in encoding and retrieval of events: what, where, and when. Proc. Natl. Acad. Sci. USA 93, 11280-11285
Nyberg, L., Persson, J., Habib, R., Tulving, E., McIntosh, A.R., Cabeza, R., Houle, S., 2000. Large scale neurocognitive networks underlying episodic memory. J. Cogn. Neurosci. 12 (1), 163-173.

Petersson, K.M., Sandblom, J., Gisselgard, J., Ingvar, M., 2001. Learning related modulations of functional retrieval networks in man. Scand. J. Psychol. 42, 197-216.

Poldrack, R.A., Wagner, A.D., Prull, M.W., Desmond, J.E., Glover, G.H., Gabrieli, J.D.E., 1999. Functional specialization for semantic and phonological processing in the left inferior prefrontal cortex. NeuroImage $10,15-35$.

Ranganath, C., Johnson, M.K., D'Esposito, M., 2000. Left anterior prefrontal activation increases with demands to recall specific perceptual information. J. Neurosci. 20, 1-5.

Ranganath, C., Paller, K.A., 1999. Frontal brain potentials during recognition are modulated by requirements to retrieve perceptual detail. Neuron 22, 605-613.

Ranganath, C., Paller, K.A., 2000. Neural correlates of memory retrieval and evaluation. Cogn. Brain. Res. 9, 209-222.

Rugg, M.D., Fletcher, P.C., Allan, K., Frith, C.D., Frackowiak, R.S.J., Dolan, R.J., 1998. Neural correlates of memory retrieval during recognition memory and cued recall. NeuroImage 8, 262-273.

Rugg, M.D., Fletcher, P.C., Chua, P.M-L., Dolan, R.J., 1999. The role of the prefrontal cortex in recognition memory and memory for source: an fMRI study. NeuroImage 10, 520-529.

Rugg, M.D., Henson, R.N.A., 2003. Episodic memory retrieval: an (eventrelated) functional neuroimaging perspective, in: Parker, A., Wilding, E., Bussey, T. (Eds.), The Cognitive Neuroscience of Memory: Encoding and Retrieval, Psychology Press, London.

Rugg, M.D., Otten, L.J., Henson, R.N.A., 2002. The neural basis of episodic memory: evidence from functional neuroimaging. Phil. Trans. R. Soc. Lond. B 357, 1097-1110.

Rugg, M.D., Wilding, E.L., 2000. Retrieval processing and episodic memory. Trends Cogn. Sci. 4 (3), 108-115.

Schacter, D.L., Tulving, E., 1994. What are the memory systems of 1994? in: Schacter, D.L., Tulving, E. (Eds.), Memory Systems, MIT Press, Cambridge, MA, pp. 1-38.

Shallice, T., Fletcher, P., Frith, C.D., Grasby, P., Frackowiak, R.S.J., Dolan, R.J., 1994. Brain regions associated with acquisition and retrieval of verbal episodic memory. Nature 368, 633-635.

Shimamura, A.P., 1995. Memory and frontal lobe function, in: Gazzaniga, M.S. (Ed.), The Cognitive Neurosciences, MIT Press, Cambridge, MA, pp. 803-813.

Snodgrass, J.G., Vanderwart, M., 1980. A standardized set of 260 pictures: norms for name agreement, image agreement, familiarity, and visual complexity. J. Exp. Psychol. Human Learn. Memory 6, 174-215.

Takahashi, E., Kenichi, O., Miyashita, Y., 2002. The role of the parahippocampal gyrus in source memory for external and internal events. Neuroreport 13, 1951-1956.

Talairach, J., Tournoux, P., 1988. Co-planar Stereotaxic Atlas of the Human Brain. Thieme, Stuttgart.

Thompson-Schill, S.L., D’Esposito, M.D., Kan, I.P., 1999. Effects of repetition and competition on activity in left prefrontal cortex during word generation. Neuron 23, 513-522.

Tulving, E., 2002. Episodic memory: from mind to brain. Annu. Rev. Physchol. 53, 1-25.

Wagner, A.D., Pare-Blagoev, E.J., Clark, J., Poldrack, R.A., 2001. Recovering meaning: left prefrontal cortex guides controlled semantic retrieval. Neuron 31, 329-338.

Wheeler, M.A., 2000. Episodic memory and autonoetic awareness, in: Tulving, E., Craik, F.I.M. (Eds.), The Oxford Handbook of Memory, Oxford University Press, Oxford, pp. 597-608.

Wilding, E.L., 2000. In what way does the parietal ERP old/new effect index recollection? Int. J. Psychophys. 35, 81-87. 